\title{
Drag reduction by the introduction of shear-free surfaces in a turbulent channel flow
}

\author{
AJAY KUMAR SOOD ${ }^{1}$, MURALI R CHOLEMARI ${ }^{1, *}$ and BALAJI SRINIVASAN ${ }^{1,2}$ \\ ${ }^{1}$ Department of Applied Mechanics, Indian Institute of Technology, New Delhi 110016, India \\ ${ }^{2}$ Present Address: Department of Mechanical Engineering, Indian Institute of Technology, Chennai 600036, \\ India \\ e-mail: murali@am.iitd.ac.in
}

MS received 29 May 2015; revised 22 March 2016; accepted 1 August 2016

\begin{abstract}
In this paper, a novel technique for drag reduction in turbulent flows is presented. The technique involves the modification of the large scales of turbulent flows and is a passive approach. The lateral transport of momentum, which is a dominant mechanism in turbulence, is attenuated by the introduction of moving shearfree surfaces (SFSes). This brings about a reduction in the drag. 2D simulations have been carried out for a turbulent channel flow using shear stress transport (SST) Reynolds-averaged Navier-Stokes (RANS) model and validated with the available experimental results. The interaction between the plates and the fluid is two way, and is enforced either by the use of a rigid body solver with moving mesh, or by considering the SFSes to be fixed at particular locations and then updating the velocities of the plates at those locations. The latter is equivalent to solving a fully developed flow in the moving mesh case. The number, shape, size and placement of the SFSes strongly influence the amount of drag reduction. The phenomenon is confirmed to be governed by a 'slow' turbulent time scale. Further, the efficacy of the method is seen to depend on the ratio of two time scales an advection time scale indicating the 'resident time' near an SFS, and the turbulent time scale. In addition, the effectiveness of the approach is improved by judicious placement of multiple SFSes in the flow.
\end{abstract}

Keywords. Drag reduction; turbulent flows; shear-free surfaces; effect on large-flow scales.

\section{Introduction}

Most flows occurring in engineering practice are turbulent. Greatly increased transport, including momentum transport, is a chief distinguishing characteristic of turbulent flows when compared with laminar flows. Due to the extremely large extent of operations involving fluid transport, even a small reduction in drag can result in huge energy savings. Thus the continuing focus of a large body of research is on the methods of reducing skin friction drag in turbulent flows. These can either be active, with active intervention into the flow by the use of additional energy, or passive, which involve no additional energy, but can involve additives. A few examples of active control strategies are the linear systems approach, the use of large-eddy break-up devices (LEBU), electromagnetic excitation and transverse wall oscillations. A summary of a few active flow control strategies and the conceptual framework for it is given in [1]. Transverse motion brought about by active (e.g., wall oscillations, traveling waves) or passive means (e.g., riblets) can also bring about a reduction in the drag. Various such mechanisms are reviewed in [2]. Drag reduction can also be brought about by modifying the near wall

*For correspondence velocity field using electromagnetic means (e.g., [3-5] and references in these works). LEBU devices attempt to reduce the drag by interfering with the transport involved with the large structures of the flow (e.g., see [6] and references therein). The theory of linear control systems approach to flow control is surveyed in [7].

Studies abound in passive control strategies as well. Passive use of compliant walls (e.g., see [8]) or the presence of riblets (e.g., [9]) is also known to cause a reduction in the drag. Passive control usually involves the introduction of one or more types of additives, usually in very small amounts into the flow. These can be long-chain polymer solutions, gaseous micro-bubbles in liquids (e.g., [10-13]), surfactants (surveyed in [14]) or rigid fibre elements (see [15] and references therein). Many of the phenomenological changes that occur in such scenarios are represented well by the case of polymer additives.

A large body of literature exists on the phenomenon of drag reduction by polymers, since its identification in 1949 $[16,17]$, also see reviews by Lumley [18] and by Berman [19]. The viscoelastic behaviour of the long-chain molecules in the solution is responsible for drag reduction in turbulent flows. But in laminar flows, the drag actually increases by the addition of the polymer [17]. There is an experimentally observed bound on the maximum amount of 
drag reduction possible. Increasing polymer concentration does not lead to drag reductions in excess of this 'maximum drag reduction asymptote' (see [20] and references therein). Although the addition of the polymers reduces the viscous dissipation, the energy is dissipated also by viscoelastic means (see [21]). Most of the passive drag reduction strategies involve additives acting at small scales, and thus the drag-reducing agent usually cannot be unmixed. This can be a disadvantage sometimes, when contamination of the transported fluid is undesirable, for example in the supply of drinking water.

In the present paper a novel method of achieving significant drag reduction in turbulent flows is introduced and studied. The technique is passive and works by modifying the large scales (greater than the inertial-range scales) of the flow. An advantage of this technique is that the additives introduced into the flow to obtain drag reduction can be recovered by mechanical separation and can be reused. No input of energy is needed to obtain drag reduction and the purity of the fluid is maintained. The amount of drag reduction that can be obtained by making use of this technique depends upon the shape, size, placement and number of the shear-free surfaces (SFSes) used.

The mechanism involved in the drag reduction can be described as follows. The technique involves the modification of the large scales of turbulent flows. The lateral transport of momentum, which is usually a dominant mechanism of turbulent transport, is attenuated by the introduction of solid surfaces moving with the flow. The introduction of these solid surfaces causes the kinematic wall-blocking effect, due to which the wall-normal velocity fluctuations are attenuated. The effect of the moving SFSes is non-local; the effect is felt over an integral scale defined using the wallnormal velocity fluctuations. The premise of the approach is that this also causes the attenuation of the wall-normal turbulent transport, in particular of momentum, and hence drag reduction is brought about. While kinematic wall blocking occurs around every immersed solid surface, simply introducing a stationary solid surface inside the flow causes additional boundary layers to form, which greatly increases the transport and hence the drag. However the SFSes move at the local speed of the flow and hence are shear-free on average. Thus, no new shear boundary layers are formed on the SFSes, and hence no counteracting effect is present for the kinematic wall blocking effect.

The proposition has been tested experimentally [22-26]. RajaVamsi [25] found significant drag reduction by adding polypropylene beads (density $=950 \mathrm{~kg} / \mathrm{m}^{3}$ ) to a gravitydriven turbulent flow in a pipe. SFSes in the form of beads instead of long strips were used for ease of implementation. The length of the pipe was $1.5 \mathrm{~m}$ and the diameter $D$ was 12 $\mathrm{mm}$. The average Reynolds number was 16,180. The flow rate increased at the same head when beads were added, indicating drag reduction. The beads were of an elliptical cross-section, approximately $1.5 \times 2.5 \times 4 \mathrm{~mm}^{3}$, which were in the middle of the inertial range for the flow. The result of his experiments is given in table 1. It was observed that the amount of drag reduction would increase with the size of the particle, suggesting that the use of thin strips as SFSes is better, which was investigated in [26]. LDPE strips of various shapes $(L / D=8-120 ; W / D=0.3-0.8)$ were used in the same set-up discussed above. The summary of these and other experimental observations is as follows. The large scales of the flow are affected by the presence of the SFSes and drag reduction is possible. The amount of drag reduction depends upon the number of SFSes present in the flow. Because of the reduced lateral transport of momentum, the velocity profile becomes more peaky when compared with the flatter turbulent profile [26]. The time scale involved is the 'slow' turbulence time scale, defined as $\tau=l / u^{\prime}$, where $l$ is a large scale of turbulence and $u^{\prime}$ is the rms fluctuation [24, 26]. In all the above works, the velocity profiles were not measured. The speed of the SFSes was measured in [26]. The presence of the drag reduction was either inferred by the increased flow rate at the same pressure difference along the pipe [25, 26] or by decreased pressure drop along the channel at the same flow rate [24]. The nature of the time scale was inferred from the observed behaviour of the SFSes.

The approach works for other turbulent transport processes as well. Analogous to the attenuation of the lateral transport of momentum reducing the drag, the reduction of lateral transport of other species would increase the transport in directions along the SFSes. This was verified in [23]. A turbulent convection in a vertical pipe with zero mean flow was considered $[27,28]$. Since the mean flow is absent, any stationary surface is shear-free. The axial flux of salt was shown to increase by as much as about $20 \%$ by the introduction of SFSes in the form of a rod placed along the pipe axis, although the rod actually blocks the area available for transport by about $12 \%$.

Several important questions remain. The velocity profiles are not known. The details of the turbulent transport, in the form of velocity fluctuations or as fluctuating pressure and velocity or as viscous transport, are unknown. The

Table 1. Summary of experiments reported by RajaVamsi [25].

\begin{tabular}{lcccccc}
\hline $\begin{array}{l}\text { S1. } \\
\text { no. }\end{array}$ & $\begin{array}{c}\text { Amount of polypropylene } \\
(\% \text { by volume })\end{array}$ & $\begin{array}{c}\text { Flow rate } \\
(\mathrm{l} / \mathrm{s})\end{array}$ & $\begin{array}{c}\text { Flow velocity } \\
(\mathrm{m} / \mathrm{s})\end{array}$ & $\begin{array}{c}\text { \% increase in } \\
\text { velocity }\end{array}$ & $\begin{array}{c}\% \text { drag } \\
\text { reduction }\end{array}$ \\
\hline $1^{*}$ & 0 & 0.153 & 1.348 & 0 & 0.031 \\
$2 *$ & 1 & 0.163 & 1.442 & 6.923 & 0.027 & 12.5 \\
\hline
\end{tabular}

* Average of 5 measurements 
dependence of the phenomenon on the Reynolds number is not known. The details of the augmentation of the effect with multiple SFSes are not known. Finally, the nature of the dependence of the effect on the shape, size and placement of the SFSes as parameters needs to be determined.

Numerical simulations of the flow would help in addressing these questions to a large extent. With simulations we can evaluate the transport terms individually, study the effect of multiple SFSes and conduct other parametric studies besides obtaining the velocity profiles. While a direct numerical simulation (DNS) or a large eddy simulation (LES) of the flow would be required for the first, the other objectives can be addressed by a suitable Reynolds-averaged Navier-Stokes (RANS) simulation.

\section{Present work}

In the present work, RANS simulations are conducted in a two-dimensional turbulent channel with SFSes. Simulations were done with a fixed mesh as well as a mesh moving with the SFSes. The fixed mesh simulations were done for SFSes that were fixed at one location, but their velocities were updated by a two-way interaction with the flow. Stationary mesh simulations are more convenient as the issues with deforming meshes are avoided. It is easy to see that the two approaches are equivalent for a very long SFS in a long channel (fully developed case). However, we will show that the two approaches are equivalent phenomenologically even for shorter SFSes. Figure 1 shows the schematics of the two approaches. Boundary conditions for the moving mesh case (figure 1a) are as follows: constant velocity inlet, pressure outlet, stationary upper and lower walls and one SFS, which is constrained to move only along the midplane of the channel with the no-slip condition on the SFS. Boundary conditions for the 'fixed mesh' case (figure 1b) are as follows: constant velocity inlet, pressure outlet, stationary upper and lower walls, with the no-slip condition on the SFSes. Three SFSes are fixed at the locations shown with their surface velocities updated every iteration by way of a two-way coupling with the flow.

The two-dimensional simulations were performed with ANSYS CFX 15 involving a geometry consisting of a rectangular channel having length to height ratio varying from 100:1 to 500:1. As discussed above, both moving and stationary meshes are used and it is shown that under certain conditions, the use of the stationary meshes is sufficient to understand the phenomenology of the problem. The shear stress transport (SST) model [29] was selected for the present work, since the simulations of stationary (with shear) and shear-free solid surfaces were simultaneously required. It was ensured that the $y^{+}$value is less than 1 so that the near wall effects were properly captured in all simulations performed. The maximum aspect ratio for the mesh used is 400. A high resolution advection scheme was used. A residual target of $10^{-6}$ (RMS) was the convergence criteria used. The interpolation schemes used were linear-linear for pressure interpolation and tri-linear for velocity interpolation. The Rhie-Chow fourth-order velocity-pressure coupling scheme was used. In the SST Model, a clip Factor with a value of 10.0 has been used as a production limiter factor. Water at $25^{\circ} \mathrm{C}$ was the working fluid in the channel. Velocity inlet with a constant velocity, lower and upper stationary walls (smooth) and pressure outlet have been used as the boundary conditions.

Two sets of simulations were done. The first set of simulations were done to identify the effects of the introduction of SFSes on the flow, as well as to demonstrate the equivalence of the moving mesh and fixed mesh simulations for a long channel with fully developed flow, and to show that the drag reduction technique is not applicable to laminar flows. A grid independence study was also done. The second set of simulations were essentially similar to

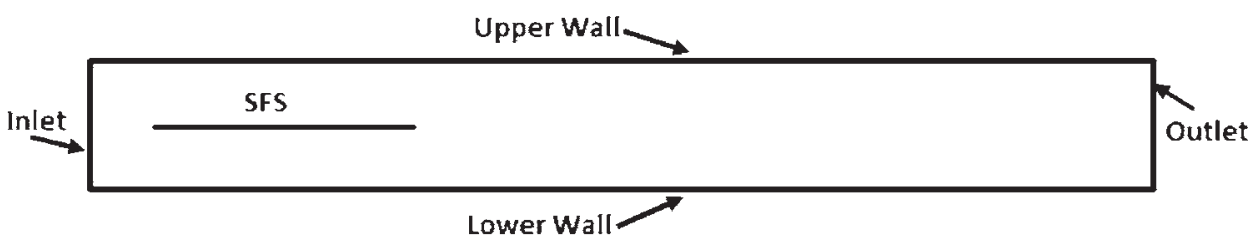

(a)

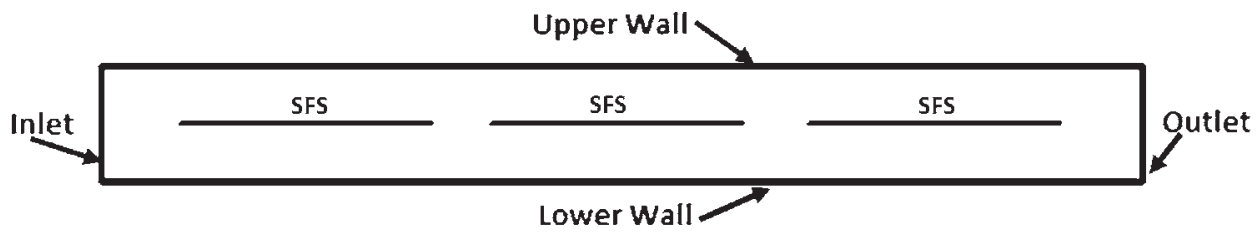

(b)

Figure 1. Equivalence of moving and stationary mesh problems. See the text for a description of boundary conditions. (a) Geometry and boundary conditions for the moving mesh problem. (b) Geometry and boundary conditions for the equivalent stationary-mesh problem with SFSes 'fixed' at particular locations. 
Table 2. 2D simulations performed with SFSes placed only along the centerline of the channel in a single row.

\begin{tabular}{|c|c|c|c|}
\hline $\begin{array}{l}\text { Sl. } \\
\text { no. }\end{array}$ & $\begin{array}{l}\text { Simulations done (with shear-free surfaces introduced only along the centreline of the } \\
\text { channel) }\end{array}$ & $\begin{array}{l}\text { Reynolds number } \\
\quad \operatorname{Re}=h U_{\text {avg }} / v\end{array}$ & $\begin{array}{l}\text { Drag } \\
\text { reduction } \\
\quad(\%)\end{array}$ \\
\hline 1 & $\begin{aligned} \text { One plate having length }= & 1 / 4 \text { th of the length of the channel introduced along the centreline } \\
& \text { of the channel } \\
& (\text { simulated as a moving mesh problem })\end{aligned}$ & 10,000 & $\sim$ zero \\
\hline 2 & $\begin{array}{l}\text { Three plates each having length }=1 / 4 \text { th of the length of the channel placed along the } \\
\text { centreline one after another with a gap of } 0.1 \mathrm{~m} \text { between consecutive plates } \\
\text { (simulated as a fixed mesh problem) }\end{array}$ & $\begin{array}{c}30,000 \\
10,000 \\
30,000 \\
1000 \text { (laminar flow) }\end{array}$ & $\begin{array}{l}\sim \text { zero } \\
\sim \text { zero } \\
\sim \text { zero } \\
\sim \text { zero }\end{array}$ \\
\hline
\end{tabular}

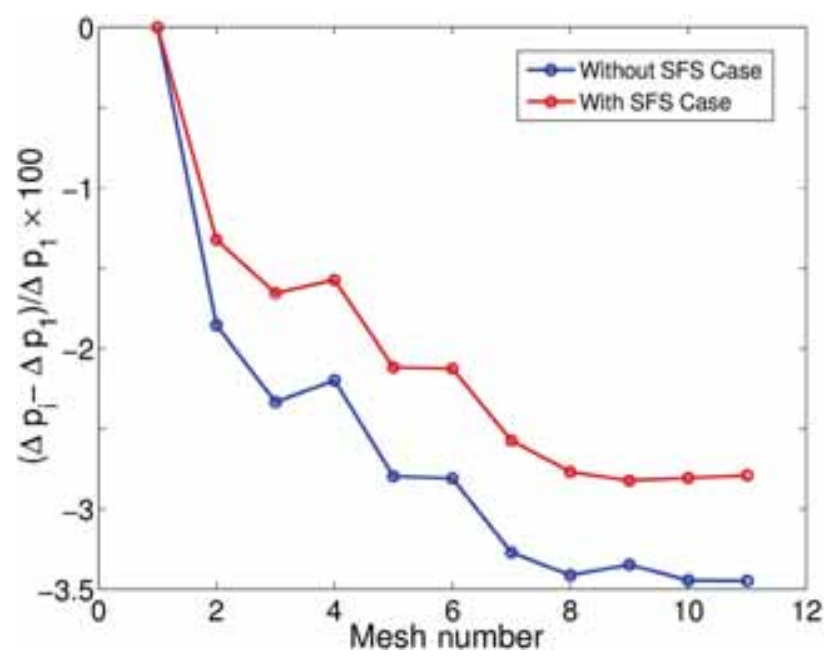

Figure 2. Grid independence study using 11 different meshes with the 1st mesh being the most coarse and the 11th being the finest mesh.

the stationary mesh simulations in the first set; however, here the size, placement and the number of the SFSes were parametrically varied. Effect of the Reynolds number was also investigated by considering simulations at two different Reynolds numbers. Multiple SFSes were placed along the height as well as along the length of the channel. These are discussed further in the next section.

\subsection{First set of simulations}

In the first set of simulations, SFSes were introduced only along the centreline of the channel. Both moving mesh and stationary mesh simulations were done in the first set. Unsteady simulations at Reynolds number (defined as $\mathrm{Re}=$ $h U_{\text {avg }} v=10,000$, where $h$ is the channel height and $U_{\text {avg }}$ is the average velocity) were done with moving mesh using a rigid body solver, allowing the plate to move only along $x$ direction and constraining all other degrees of freedom of the plate. The geometry used for moving mesh case is shown in figure $1 \mathrm{a}$. The length of the channel was varied from 2 to $10 \mathrm{~m}$ to study how long it takes for the flow to become fully developed and hence conclude on the adequacy of the 'stationary' plate simulations (see below). The mass of the SFS was $0.0001 \mathrm{~kg}$. The typical length of the SFSes was $50 \mathrm{~cm}$ with a gap of $10 \mathrm{~cm}$. This was chosen to simulate the LDPE sheets used as SFSes in [26]. Unless otherwise mentioned, the height of the channel was $0.02 \mathrm{~m}$.

Also, unsteady and steady state simulations at Reynolds number of 10,000 and 30,000 were run using a stationary mesh (fixed SFS) but with the velocity of the SFS updated according to the shear force acting on it. Conceptually it is like an SFS appearing at a point, moving with the flow and disappearing at a downstream point. The velocities of the SFSes are updated every iteration based on the net force acting on the plate in the direction of flow. The updated velocity is calculated as follows:

$$
V=U+a t
$$

where $V$ and $U$ are the velocities of the plate at the current iteration and at the previous iterations, respectively, $F$ is the net force acting on the plate along the direction of flow, $m$ is the mass of the plate and $a$ is the acceleration of the plate $(=F / m)$. For transient simulations $t$ is the size of time step, while in case of steady state simulations $t$ was taken to be the same as the time step size used in transient problems (on the order of $1 \mathrm{e}-05 \mathrm{~s}$ ). In addition a simulation for a laminar flow case (Reynolds number $=1000$ ) was also done. The summary of the simulations in set 1 is shown in table 2.

Figure 2 shows the result of the grid independence study. Both with SFS and without SFS cases are considered, but only the stationary mesh simulations are considered. The figure shows that beyond the sixth mesh, further refinement yields only a small improvement, and hence meshes of this refinement are chosen for the present simulations. At this level of refinement, the $y^{+}$value near the walls is slightly less than unity.

\subsection{Second set of simulations}

In the second set of simulations, multiple SFSes were placed along the height as well as along the length of the 


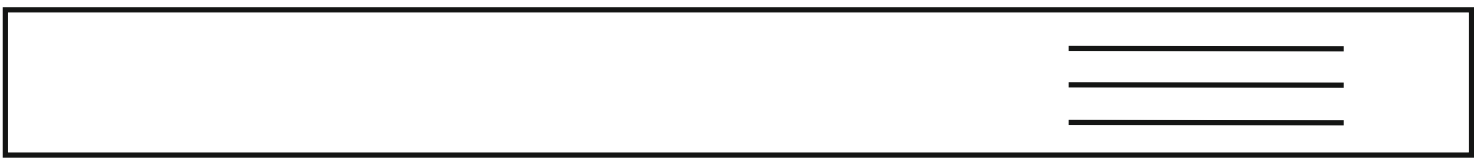

Case 1

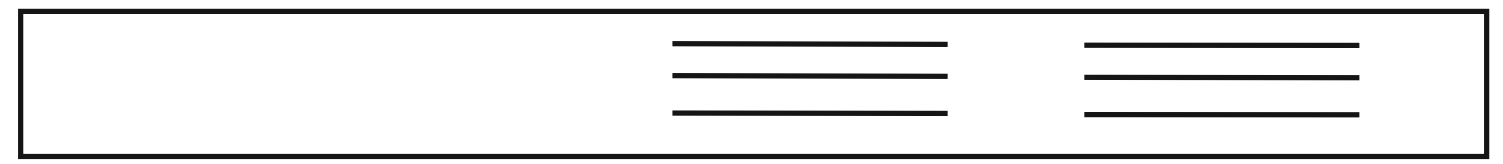

Case 2

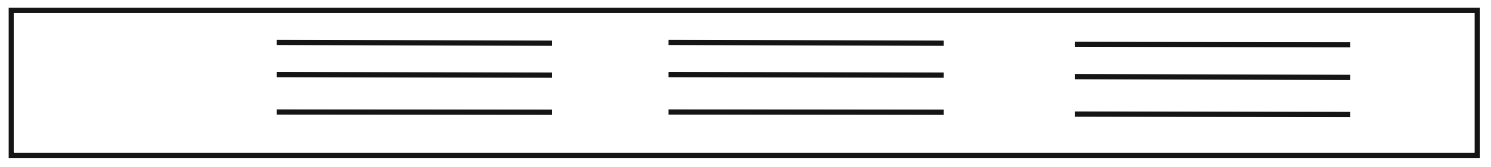

\section{Case 3}

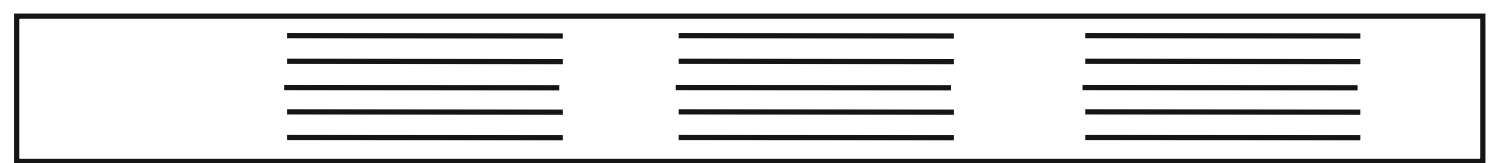

Case 4

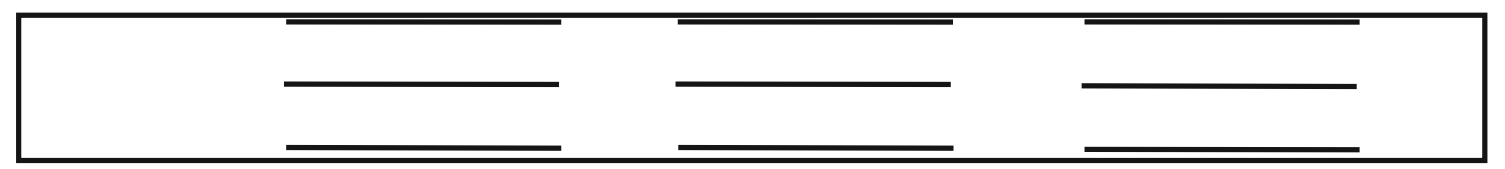

\section{Case 5}

Figure 3. Schematics for various cases considered in table 3. Flow comes in from the left side and goes out of the right side. Upper and lower surfaces are walls of the channel. Other boundaries are similar to those in figure 1.

Table 3. 2D simulations performed with SFSes placed along the height as well as along the length of the channel.

S1. Simulations done (with shear-free surfaces introduced along the centreline as well as along Reynolds number Re Drag no. the height of the channel and simulated as fixed mesh problems) reduction

\begin{tabular}{|c|c|c|c|}
\hline no. & the height of the channel and simulated a & $=h U_{\mathrm{avg}} / v$ & $(\%)$ \\
\hline \multirow[t]{2}{*}{1} & 3 plates (length of each plate $=0.5 \mathrm{~m}$ ) placed symmetrically in 3 rows and 1 column inside & 10,000 & 1.0 \\
\hline & the channel at $0.1 \mathrm{~m}$ from the exit of the channel (figure $3 \mathrm{a}$ ) & 30,000 & 0.4 \\
\hline \multirow[t]{2}{*}{2} & 6 plates (length of each plate $=0.5 \mathrm{~m}$ ) placed symmetrically in 3 rows and 2 columns inside & 10,000 & 6.9 \\
\hline & the channel (figure $3 b$ ) & 30,000 & 4.9 \\
\hline \multirow[t]{2}{*}{3} & 9 plates (length of each plate $=0.5 \mathrm{~m}$ ) placed symmetrically in 3 rows and 3 columns inside & 10,000 & 15.3 \\
\hline & the channel (figure $3 \mathrm{c}$ ) & 30,000 & 11.6 \\
\hline \multirow[t]{2}{*}{4} & 15 plates placed (length of each plate $=0.5 \mathrm{~m}$ ) placed symme trically in 5 rows and 3 & 10,000 & 28.7 \\
\hline & columns inside the channel (figure $3 \mathrm{~d}$ ) & 30,000 & 24.6 \\
\hline \multirow[t]{2}{*}{5} & 9 plates placed in 3 rows and 3 columns with 1 row placed at $1 \mathrm{~mm}$ from the upper wall, 1 & 10,000 & 48.5 \\
\hline & $\begin{array}{c}\text { row placed at } 1 \mathrm{~mm} \text { from the lower wall and } 1 \text { row at the middle of the channel } \\
\text { (figure } 3 \mathrm{e} \text { ) }\end{array}$ & 30,000 & 50.9 \\
\hline
\end{tabular}

channel. Distance between consecutive plates along the length of channel is $0.1 \mathrm{~m}$ and distance between plates along the height of the channel is variable. The mass of each plate is $0.0001 \mathrm{~kg}$. The schematics of the cases considered are in figure 3. The summary of the cases is shown in table 3 . Velocity of each plate is updated at every iteration based on the net force acting on each plate in the direction of flow (see Eq. (1)). 


\section{Results and discussion}

The evolution of the flow and the equivalence of the moving mesh and stationary mesh cases are discussed first. Figure 4 shows the velocity profiles obtained in set 1 (moving mesh case). A single SFS of $0.5 \mathrm{~m}$ length was introduced at the inlet and allowed to move with the flow. The presence of SFS attenuates the wall-normal fluctuations and hence reduces the turbulent transport (of momentum) along the wall-normal direction. This reduces the friction to the flow and the flow starts to move faster locally around the SFS. Since the kinematic wall blocking is felt over an integral scale in terms of the wall-normal fluctuations, the effect of the SFS in the velocity profile also is expected to be felt over a similar distance. However, because of continuity, the slope near the stationary walls should reduce, bringing about a net drag reduction. This is seen in figure 4 , where the velocity profiles at $5 \mathrm{~cm}$ from the leading edge of the SFS, when the leading edge is $10 \mathrm{~cm}$ from the channel exit, are plotted. Also plotted is the velocity profile in the absence of the plate. The velocity profile is significantly affected by the presence of the SFS; the velocity profile has become more peaky at the central region near the SFS, the change extending to about one integral scale on either side of the SFS. This implies that the SFS is moving faster compared with the fluid in its absence as observed in [26]. By continuity, a small reduction of the slope of the velocity profile is also seen near the walls. This reduction in the slope is the indication of the drag reduction. Also, the plate is being accelerated by the flow, as seen by the dip in the velocity at the SFS. Clearly, the profile is still evolving at $10 \mathrm{~m}$. As inferred in [26], the phenomenon is seen to be of the 'slow' turbulent time scale.

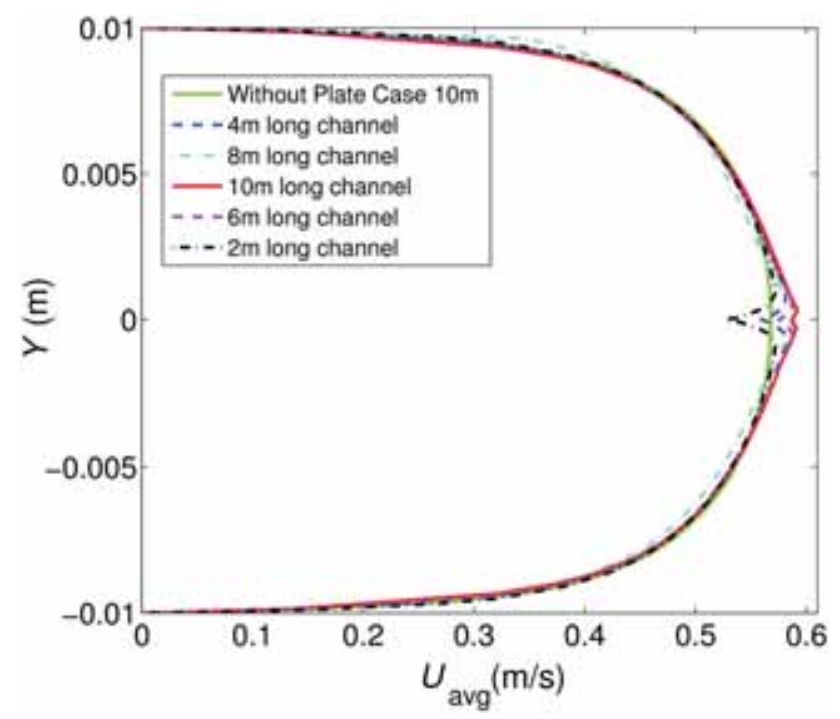

Figure 4. Velocity profiles for moving mesh cases (2, 4, 6, 8 and $10 \mathrm{~m}$ long channel) having one SFS along the centreline and the case without any SFS (10 m long channel).
Thus, while drag reduction is brought about, quite early after the introduction of the SFS, it will become truly fully developed only at large distances. However, the salient features of the drag reduction are very similar in both the fully developed as well as developing cases; hence the fully developed case is considered further in the fixed mesh simulations.

Results from the stationary mesh simulations from set 1 are shown in figure 5. Here the location of the SFS is fixed, but the SFS has a velocity determined by its two-way interaction with the flow. Three SFSes, each of length 50 $\mathrm{cm}$ with a gap of $10 \mathrm{~cm}$ between them, were placed in the flow. The leading edge of the last SFS was at $10 \mathrm{~cm}$ from the exit. This arrangement was necessary to simulate a fully developed situation. Since the flow itself would evolve from the inlet, having a single SFS would always have a drag due to the velocity evolution. However, since the flow time scale is the slow turbulent time scale, the effects of the SFS would persist mostly in the small gap of $10 \mathrm{~cm}$ between the SFSes, thus simulating the fully developed situation without the additional drag on the SFS. Figure 5 shows the velocity profile at $5 \mathrm{~cm}$ from the leading edge of the last SFS, when the leading edge is $10 \mathrm{~cm}$ from the channel exit. The profile clearly shows the effect of the SFS: the velocity profile has become more peaky over a region scaling like an integral scale, and that, by continuity, the velocity gradients at the walls have reduced, bringing about the drag reduction. Further, the profile is very close to the $10 \mathrm{~m}$ channel case from set 1 , indicating the equivalence of the two approaches for a long channel. Although the velocity profiles in the stationary and moving mesh cases are not exactly identical, the salient features of the phenomenon are identical in the two approaches. Hence we

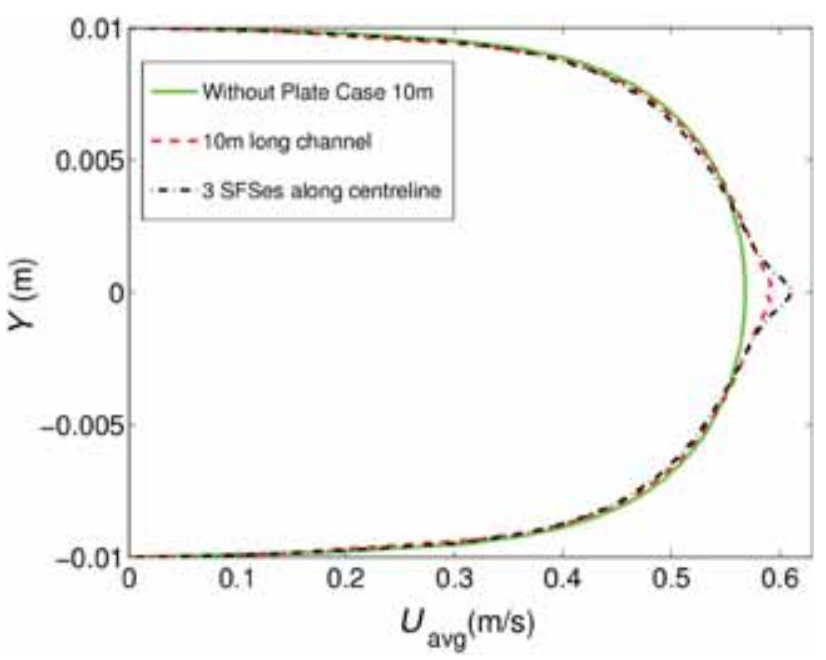

Figure 5. Comparison of velocity profiles for moving mesh cases (10 $\mathrm{m}$ long channel) having one SFS along the centreline, the case without SFS and the stationary mesh case (3 SFSes along the centreline of the channel $2 \mathrm{~m}$ long). 
(a)

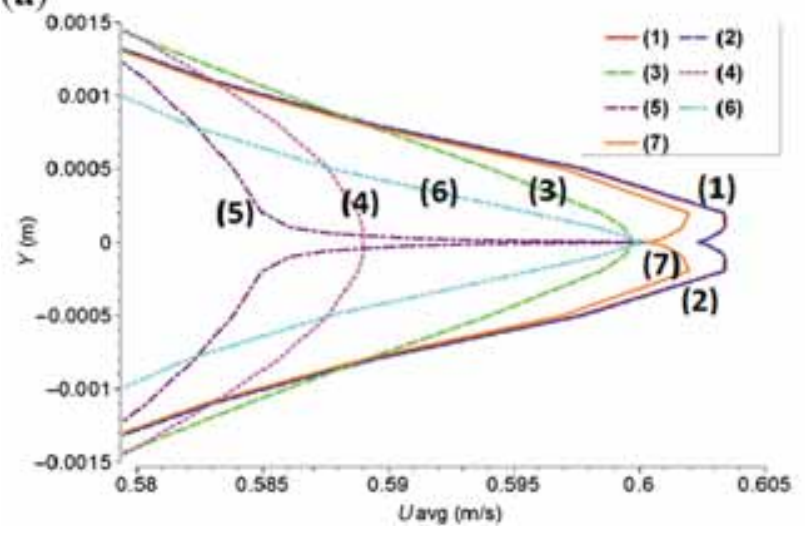

(b)

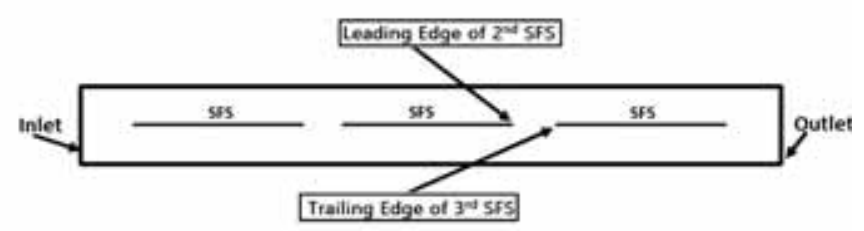

Figure 6. (a) (1) Velocity profiles shown in the figure correspond to locations $5 \mathrm{~cm}$ before the leading edge of $2 \mathrm{nd}$ SFS, (2) leading edge of 2nd SFS, (3) $7 \mathrm{~cm}$ after leading edge of 2nd SFS, (4) $14 \mathrm{~cm}$ after leading edge of 2nd SFS, (5) trailing edge of 3rd SFS, (6) $5 \mathrm{~cm}$ after trailing edge of 3rd SFS and (7) leading edge of 3rd SFS (see figure 6b). The SFSes are $50 \mathrm{~cm}$ long. (b) Region considered for the plot in figure 6 a.

use stationary mesh simulations to study the effect of SFSes towards drag reduction in the subsequent sections.

Figure 6a shows the imposing and relaxing of the kinematic wall-blocking effect as a function of distance. Fixed plate simulations from set 1 were considered. The velocity profiles starting from the leading edge of the second plate till the leading edge of the third plate are shown (see figure 6b). The imposing and relaxing of the kinematic wall blocking effect is seen in the variation of the velocity profiles along with distance along the channel. Effectively, the distance along the channel corresponds to the time of residence of a fluid particle adjacent to an SFS, since the fluid particle is essentially advected by the side of the SFS. As can be seen, the effect is reduced to about half by $14 \mathrm{~cm}$ after the SFS. This corresponds to $0.24 \mathrm{~s}$ at a mean speed of $0.5 \mathrm{~m} / \mathrm{s}$. The 'slow' turbulence time scale can be estimated using the channel width $h$ and the kinetic energy $k$ as $\tau=l /$ $u^{\prime}=h /(2 k / 3)^{1 / 2} \sim 0.3 \mathrm{~s}$. The kinetic energy $k$ is measured in the absence of the SFSes. This is indeed comparable to the observed time scale. This shows that the phenomenon is indeed of the 'slow' turbulent time scale, as observed in [26]. The imposing of the phenomenon has a similar behaviour. The significance of this is that when multiple smaller SFSes are moving with small separation distances between them, the effect is augmented, as long as the sum of the lengths of the SFSes along the flow is greater than the total length of the space where there is no SFS. This factor would be important for drag reduction involving multiple smaller SFSes, like in $[22,25,26]$, although it is expected that the size of the SFS needs to be at least in the middle of the inertial range for the effect to be significant.

Figure 7 shows that in case of laminar flows, the velocity profile does not change when the SFS is introduced along the centreline of the channel. Also no drag reduction takes place (no change in the pressure drop along the channel upon the

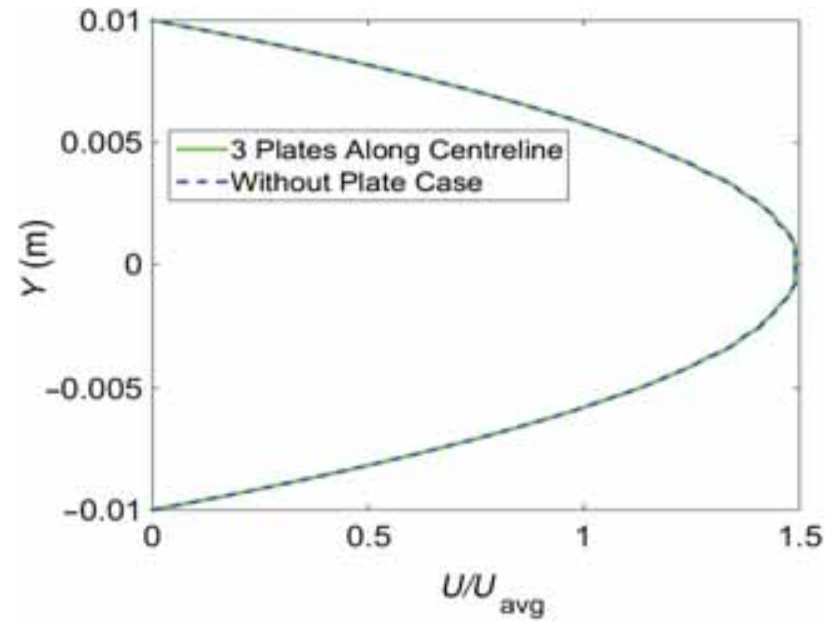

Figure 7. Laminar flow velocity profile comparison at $0.2 \mathrm{~m}$ from the exit of channel (with and without the presence of SFSes inside the channel).

introduction of SFSes). Since, in laminar flows, the pressure fluctuations are not responsible for momentum transfer, and no velocity fluctuations exist, SFSes have no effect. The attenuation of the wall-normal velocity fluctuations due to SFSes occurs only in turbulent flows and hence the attenuation of the momentum transport is responsible for the drag reduction in case of turbulent flows only.

Table 2 summarises the simulations in set 1 (done with SFSes introduced only along the centreline of the channel). In all cases, there is a clear effect of the SFSes: the velocity profile is significantly affected. However, the drag reduction is not significant. This is because, the effect of the SFS is local, and the reduction of the slope at the wall by continuity is not significant. Hence it is necessary to introduce multiple SFSes along the channel cross-section to bring 


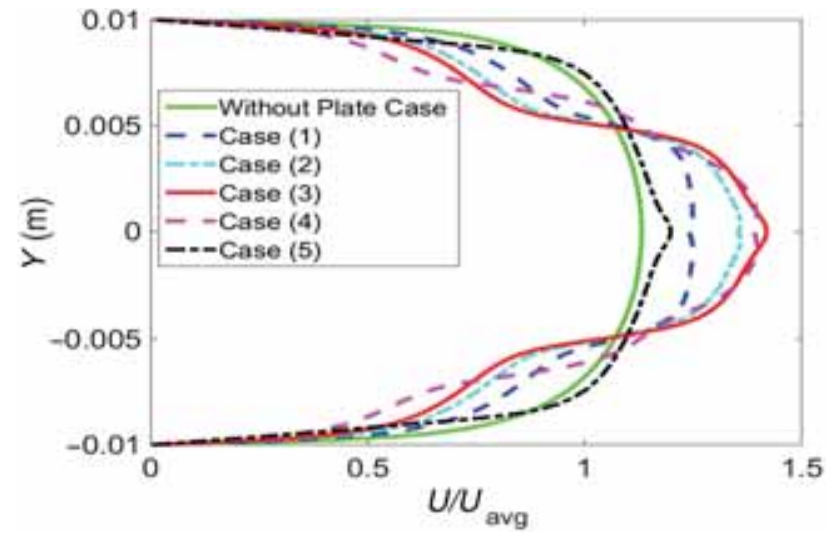

Figure 8. Comparison of velocity profiles at a location $0.2 \mathrm{~m}$ from the exit at $\mathrm{Re}$ around 10,000 for the cases considered in figure 4 and table 3 (cases 1-5).

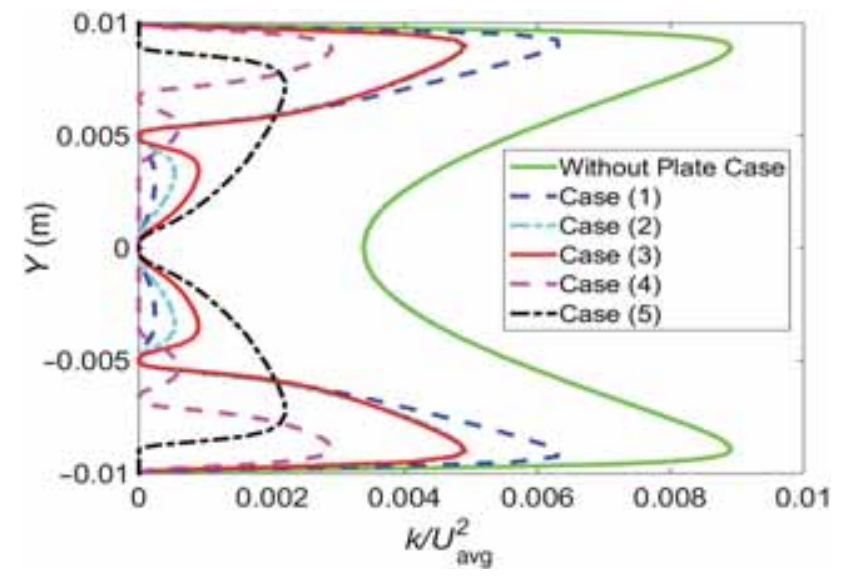

Figure 9. Comparison of turbulent kinetic energy at a location $0.2 \mathrm{~m}$ from the exit at $\mathrm{Re}$ around 10,000 for the cases considered in figure 4 and table 3 (cases 1-5).

about a significant change near the wall. This is done in the second set of simulations. Also in case of laminar flow, no change is seen in the profile because of the presence of the SFS, as expected.

Results of the second set of simulations are discussed next. The results are summarised in table 3 . Figure 8 shows the effect of the distributed placement of the SFSes. Since the kinematic wall blocking effect is local (only up to an integral scale), placing more SFSes would augment the effect, as seen in figure 8 when compared with figure 5 . It is clearly seen that the reduction of the lateral transport of momentum reduces the drag and makes the velocity profile more peaky. The location of the SFS can be further optimised as discussed below. However, increasing the number of SFSes would definitely cause more drag reduction.

Figure 9 shows the comparison of turbulent kinetic energy at a location $0.2 \mathrm{~m}$ from the exit of the channel for the cases considered in figure 3. It shows that the turbulent kinetic energy decreases significantly as the SFSes are

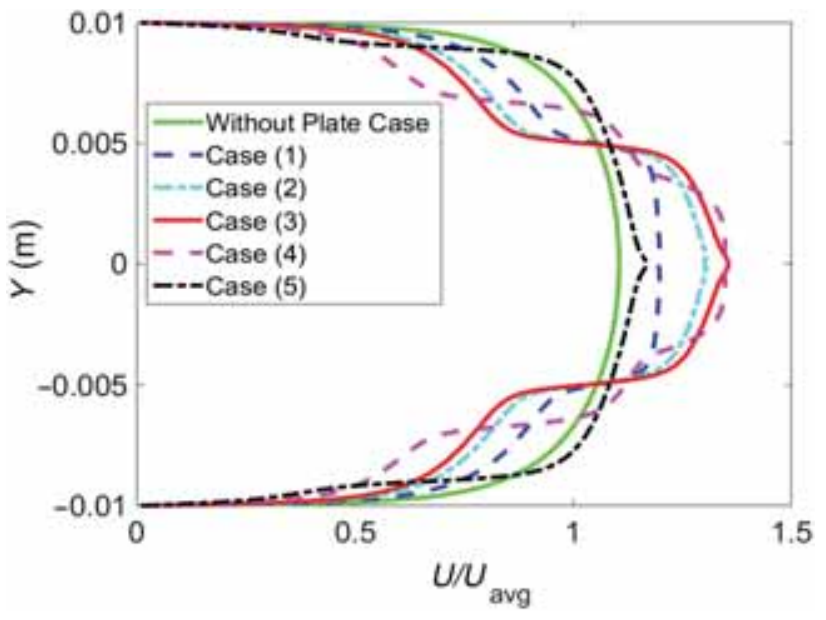

Figure 10. Comparison of velocity profiles at a location $0.2 \mathrm{~m}$ from the exit at $\mathrm{Re}$ around 30,000 for the cases considered in figure 4 and table 3 (cases 1-5).

introduced. The total amount of kinetic energy within the channel reduces, not just locally near the SFS. The kinetic energy decreases even more as more and more SFSes are introduced in the channel.

It can be observed from figure 8 and from table 3 that as the distance of the plate from the wall decreases, drag reduction increases. Further studies are required to optimise this distance. For case (e) in figure 3, three plates have been introduced, one each at $1 \mathrm{~mm}$ from both upper and lower walls and one along the central axis. There is a drag reduction of about $50 \%$. In all cases, drag reduction is accompanied by greatly reduced turbulent kinetic energy (figure 9).

Figure 10 shows the velocity profiles at a location $0.2 \mathrm{~m}$ from the exit at Re around 30,000 for the cases considered in figure 3. The higher Reynolds number is achieved in the same channel with a higher mean velocity of $1.5 \mathrm{~m} / \mathrm{s}$. Thus, the residence time of the fluid adjacent to the SFS is reduced at the higher Reynolds number, and hence the kinematic wall-blocking effect is slightly reduced. This is indicated by the slight reduction in the extent of influence of the SFS as seen in the profiles in figure 10.

Figure 11 shows the turbulent kinetic energy at a location $0.2 \mathrm{~m}$ from the exit at Re around 30,000 for the cases considered in figure 7. Here again, the turbulent kinetic energy is significantly reduced by the presence of the SFSes. As in the case of the velocity profiles, the turbulent kinetic energy is affected to a slightly lesser extent as the residence time is reduced.

\subsection{Effect of the Reynolds number and the time scale of the phenomenon}

To understand the effect of the Reynolds number and the time scale of the phenomenon, the following was done. The case of the Reynolds number of 30,000 from set 2 was 


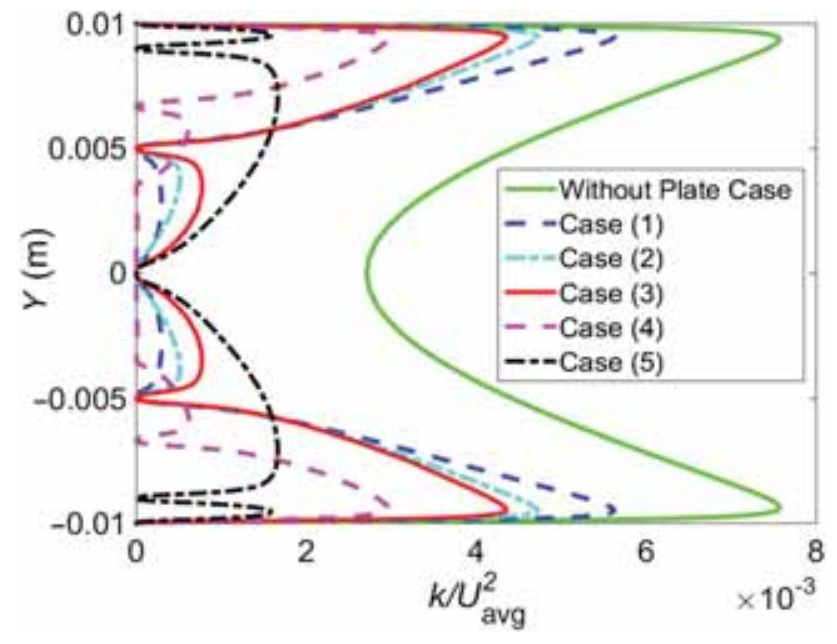

Figure 11. Comparison of turbulent kinetic energy at a location $0.2 \mathrm{~m}$ from the exit at Re around 30,000 for the cases considered in figure 4 and table 3 (cases 1-5).

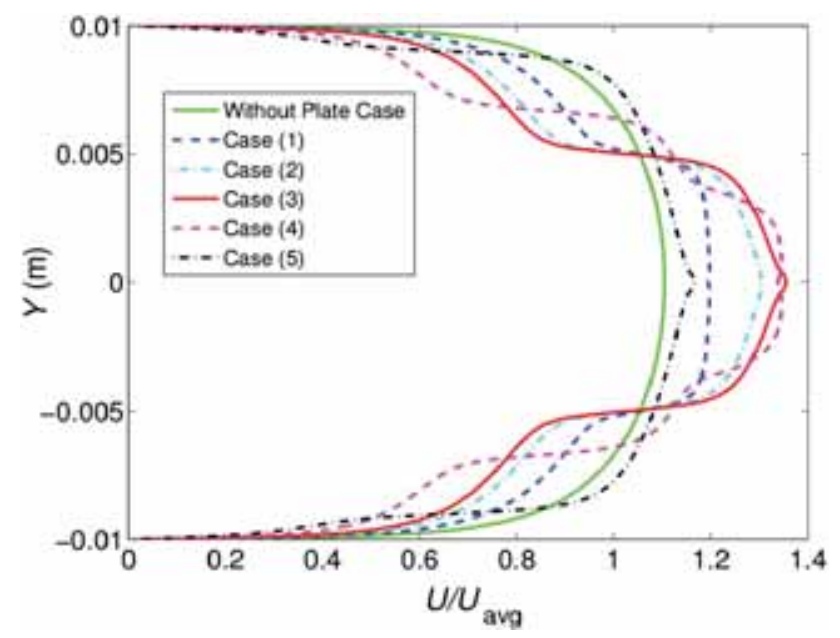

Figure 12. Velocity profiles scaled by the average velocity ( $U_{\text {avg }}$ $=1.5 \mathrm{~m} / \mathrm{s}$ ) at a location $0.2 \mathrm{~m}$ from the exit at Re around 30,000 for the cases considered in figure 3 and table 4 (cases 1-5).

simulated with the same physical dimensions of the channel as above, but with varying velocities and the viscosity of the fluid correspondingly varied. In the earlier cases, the Reynolds number of 10,000 was with an average flow velocity of $0.5 \mathrm{~m} / \mathrm{s}$ (figures 8 and 9) and the Reynolds number of 30,000 was achieved with an average flow velocity of $1.5 \mathrm{~m} / \mathrm{s}$ (figures 10 and 11) with the fluid and the channel dimensions being kept the same. The Reynolds number of 30,000 was simulated again with an average velocity of $0.5 \mathrm{~m} / \mathrm{s}$ but with the kinematic viscosity reduced to $1 / 3$ times its earlier value, and again with an average velocity of $0.05 \mathrm{~m} / \mathrm{s}$ but with the kinematic viscosity reduced to $1 / 30$ times its earlier value. The channel height was not changed. The mean velocity profiles scaled with the average velocity and the turbulent kinetic energy profiles scaled with the average velocity squared are presented

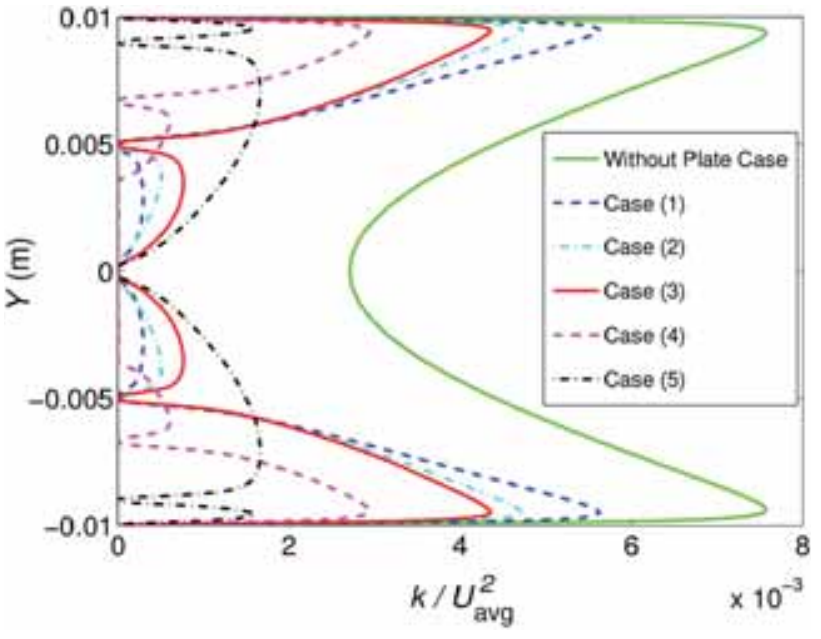

Figure 13. Turbulent kinetic energy scaled by $\left(U_{\text {avg }}\right)^{2}$ at a location $0.2 \mathrm{~m}$ from the exit at $\mathrm{Re}$ around 30,000 for the cases considered in figure 3 and table 4 (cases $1-5$ ), $U_{\text {avg }}=1.5 \mathrm{~m} / \mathrm{s}$.

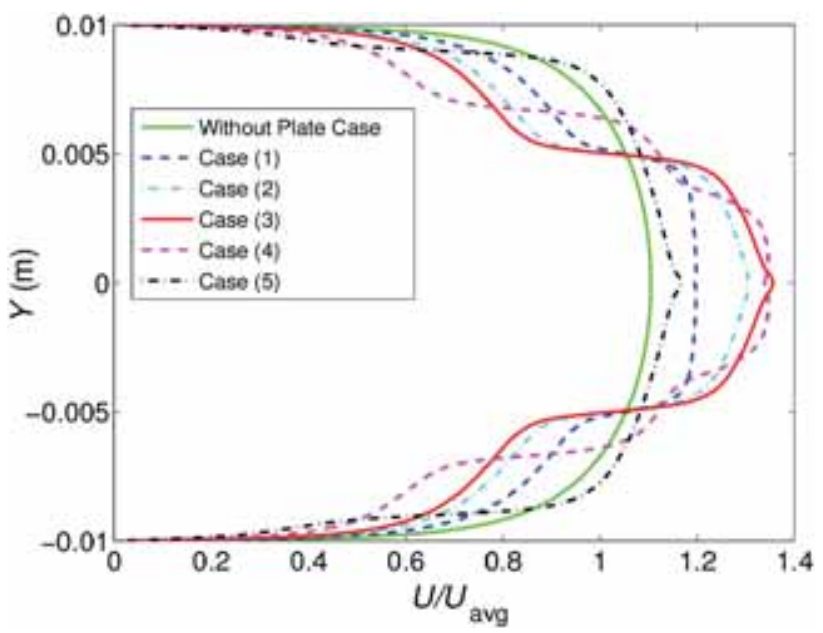

Figure 14. Velocity profiles scaled by the average velocity $\left(U_{\text {avg }}\right.$ $=0.5 \mathrm{~m} / \mathrm{s}$ ) at a location $0.2 \mathrm{~m}$ from the exit at Re around 30,000 corresponding to the cases considered in figure 3 and table 4 (cases 1-5).

for the cases with Reynolds number $=30,000$ in figures $12-$ 17. The figures for the scaled mean velocity (figures 12, 14, and 16) are identical. The figures for the scaled turbulent kinetic energy (figures 13, 15, and 17) are also identical. Also shown in figures 18 and 19 are the scaled velocity profiles and the scaled turbulent kinetic energy profiles for the case of Reynolds number $=10,000$. There is some change in the scaled profiles of velocity and the turbulent kinetic energy between $\operatorname{Re}=10,000$ and $\operatorname{Re}=30,000$ cases. In summary, the phenomenon is dependent on the Reynolds number. When the mean velocity is kept the same, but the Reynolds number is made different, the drag reduction and the profiles of velocity and the turbulent kinetic energy are different. 


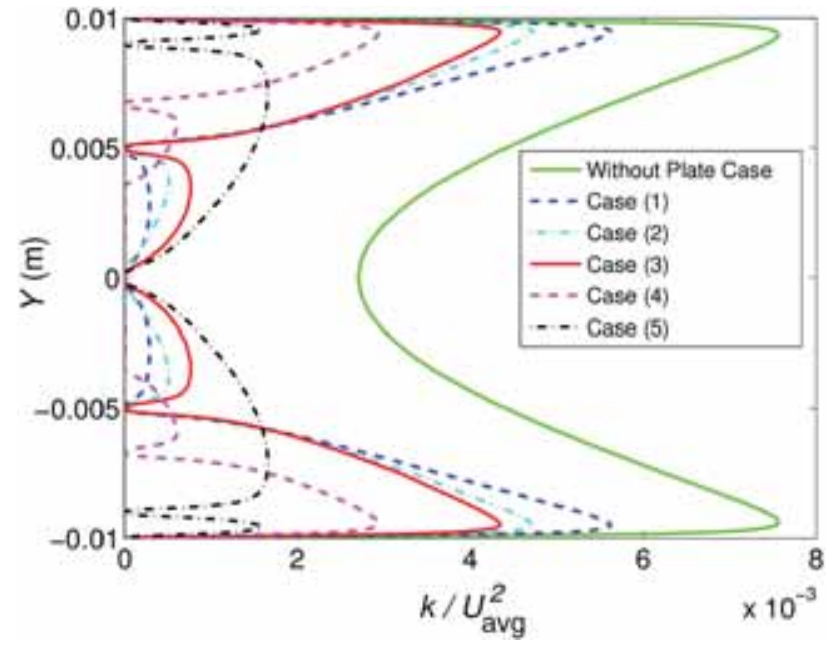

Figure 15. Turbulent kinetic energy scaled by $\left(U_{\text {avg }}\right)^{2}$ at a location $0.2 \mathrm{~m}$ from the exit at $\mathrm{Re}$ around 30,000 corresponding to the cases considered in figure 3 and table 4 (cases $1-5$ ), $U_{\text {avg }}=0.5$ $\mathrm{m} / \mathrm{s}$.

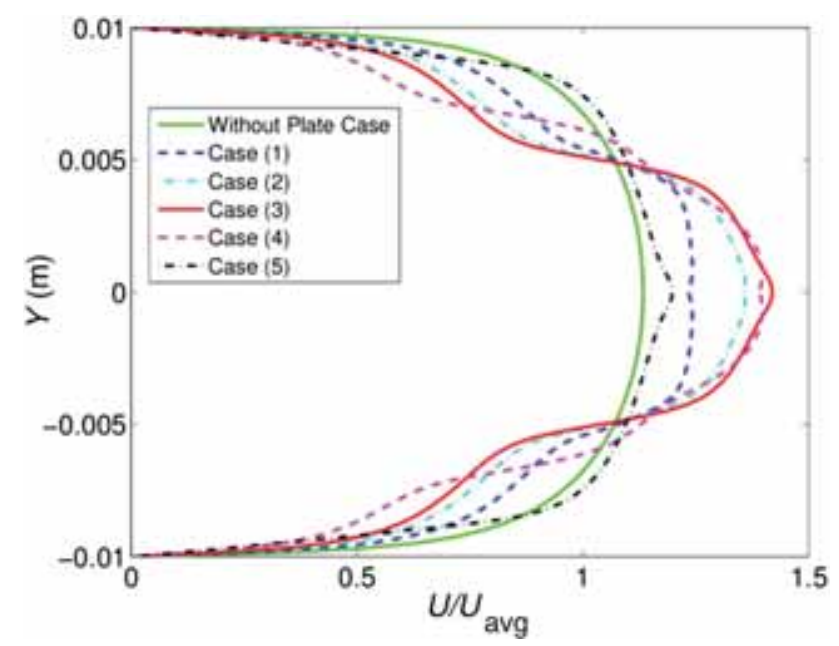

Figure 16. Velocity profiles scaled by the average velocity $\left(U_{\text {avg }}\right.$ $=0.05 \mathrm{~m} / \mathrm{s}$ ) at a location $0.2 \mathrm{~m}$ from the exit at Re around 30,000 corresponding to the cases considered in figure 3 and table 4 (cases 1-5).

This can be understood in the following way: $1 / u^{\prime}(=h /(2 /$ $3 k)^{1 / 2}$ ) is the time scale of the effect (the 'slow' turbulent time scale). Here $h$ is the channel width, which is a large scale of the flow. Now the imposing or the relaxing of the phenomenon depends on the resident time of a fluid particle adjacent to an SFS. Hence the ratio of two time scales becomes important: the advection time scale $L / U_{\text {avg }}$, and the turbulent time scale $h / u^{\prime}$. Here $L$ is the length of an SFS. However, $u^{\prime} / U_{\text {avg }}$ is approximately constant for a given Reynolds number, as discussed below. Hence the total time available for the phenomenon to take effect is about the same for a given Reynolds number; $\left(L / U_{\mathrm{avg}}\right) /\left(h / u^{\prime}\right)$ is approximately constant for a given Reynolds number.

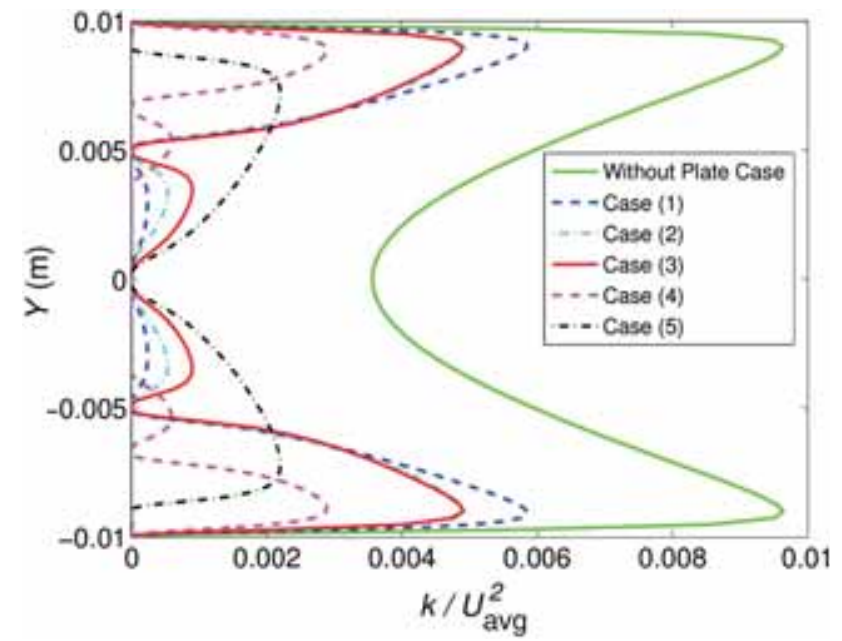

Figure 17. Turbulent kinetic energy scaled by $\left(U_{\text {avg }}\right)^{2}$ at a location $0.2 \mathrm{~m}$ from the exit at Re around 30,000 corresponding to the cases considered in figure 3 and table 4 (cases 1-5), $U_{\text {avg }}=$ $0.05 \mathrm{~m} / \mathrm{s}$.

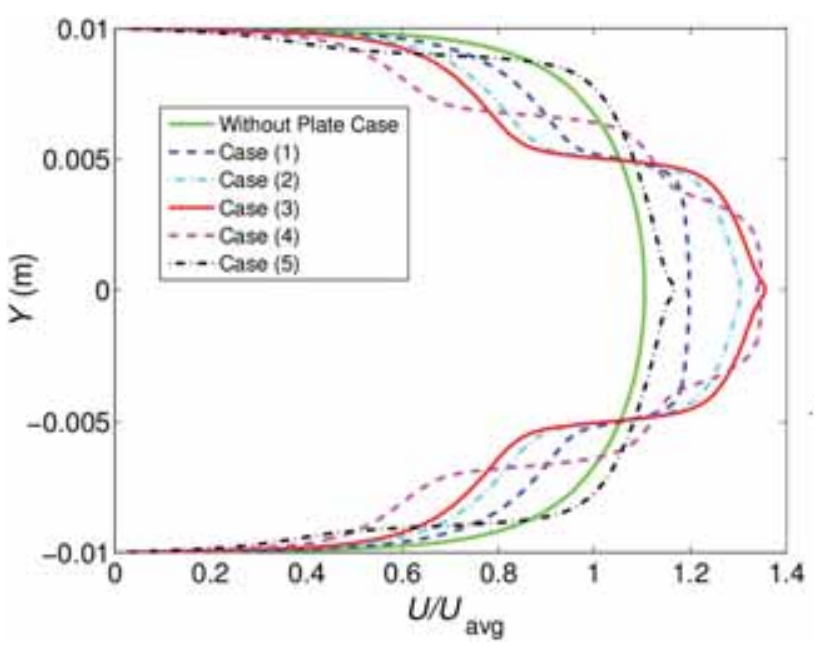

Figure 18. Velocity profiles scaled by the average velocity $\left(U_{\text {avg }}\right.$ $=0.5 \mathrm{~m} / \mathrm{s}$ ) at a location $0.2 \mathrm{~m}$ from the exit at Re around 10,000 for the cases considered in figure 3 and table 4 (cases 1-5).

Hence, changing the velocity but keeping the Reynolds number the same with the geometric parameters being the same has no change in the phenomenon, as seen in figures 12-17.

The fact that $u^{\prime} / U_{\text {avg }}$ is approximately constant for a given Reynolds number can be seen in the following way. Figure 7.10 of Pope [30] shows the skin friction $C_{f}$ as a function of the Reynolds number. $C_{f}=\rho u_{\tau}^{2} /\left((1 / 2) \rho U^{2}\right)$, decreasing as a function of the Reynolds number. Here $u_{\tau}$ is the friction velocity. In wall bounded flows, $u^{\prime} / u_{\tau}$ and $v^{\prime} / u_{\tau}$ have roughly the same profiles across a wide Range of Reynolds numbers (see figure 7.19 of Pope [30]); hence $u^{\prime}$ and $v^{\prime}$ behave similarly with the Reynolds number 


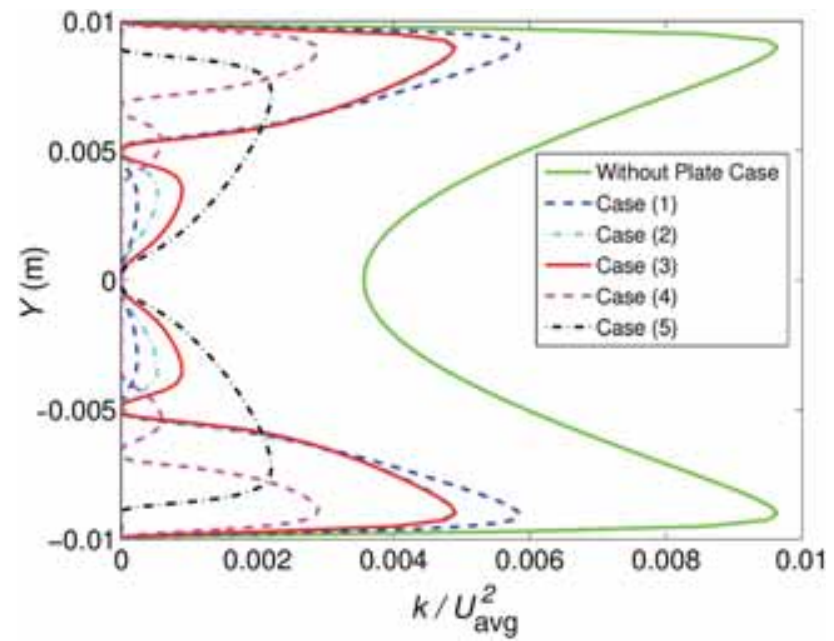

Figure 19. Turbulent kinetic energy scaled by $\left(U_{\text {avg }}\right)^{2}$ at a location $0.2 \mathrm{~m}$ from the exit at Re around 10,000 for the cases considered in figure 3 and table 4 (cases $1-5$ ), $U_{\text {avg }}=0.5 \mathrm{~m} / \mathrm{s}$.

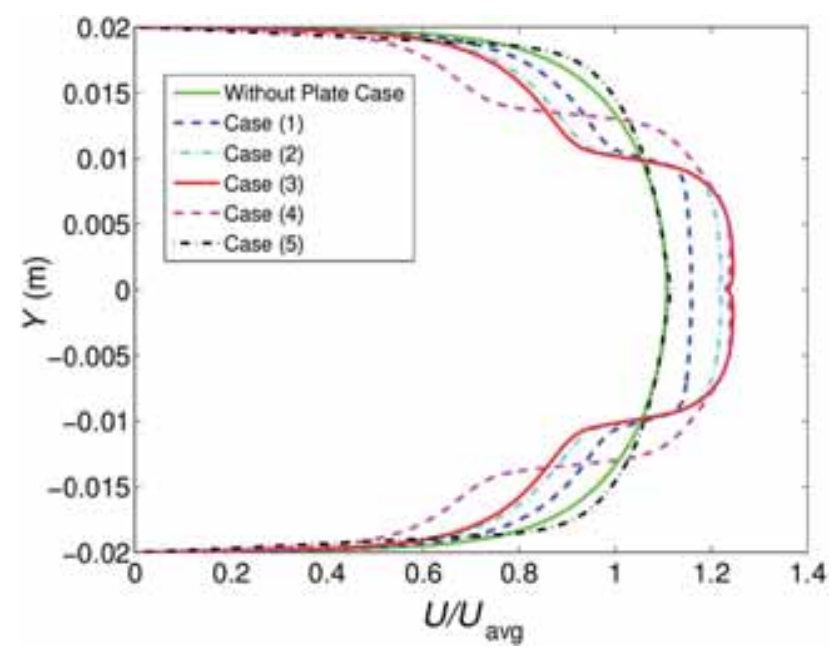

Figure 20. Velocity profiles scaled by the average velocity $\left(U_{\text {avg }}\right.$ $=0.75 \mathrm{~m} / \mathrm{s}$ ) at a location $0.2 \mathrm{~m}$ from the exit at Re around 30,000 for the cases considered in figure 3 and table 4 (cases 1-5).

(decrease with the Reynolds number); $u^{\prime} / U$ at $\operatorname{Re}=30,000$ is about $80 \%$ of the same at $\operatorname{Re}=10,000$. This is reflected in the fact that the drag reducing effect does not change if the Reynolds number is kept the same, but the velocity is varied and the geometry is kept the same. Also it explains the changes in the profiles of the mean velocity and the turbulent kinetic energy around the SFS, and the change in the drag reduction with the Reynolds number.

Continuing thus, doubling $h$, halving $U_{\text {avg }}$ and keeping $L$ and the viscosity the same would halve the ratio $\left(L / U_{\text {avg }}\right) /$ $\left(h / u^{\prime}\right)$. Thus the resident time would decrease relative to the turbulent time scale and hence decrease the drag reduction

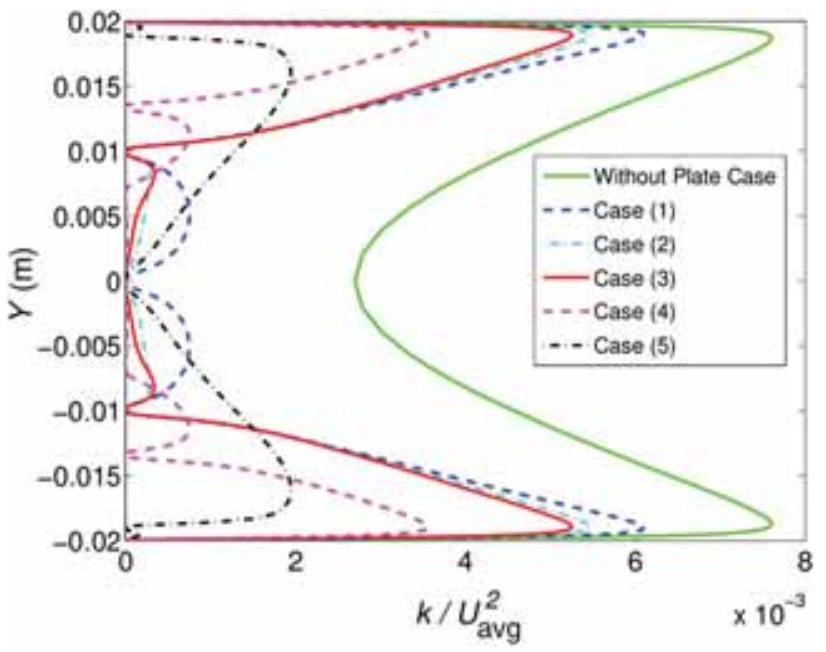

Figure 21. Turbulent kinetic energy scaled by $\left(U_{\text {avg }}\right)^{2}$ at a location $0.2 \mathrm{~m}$ from the exit at $\mathrm{Re}$ around 30,000 for the cases considered in figure 3 and table 4 (cases $1-5$ ), $U_{\text {avg }}=0.75 \mathrm{~m} / \mathrm{s}$.

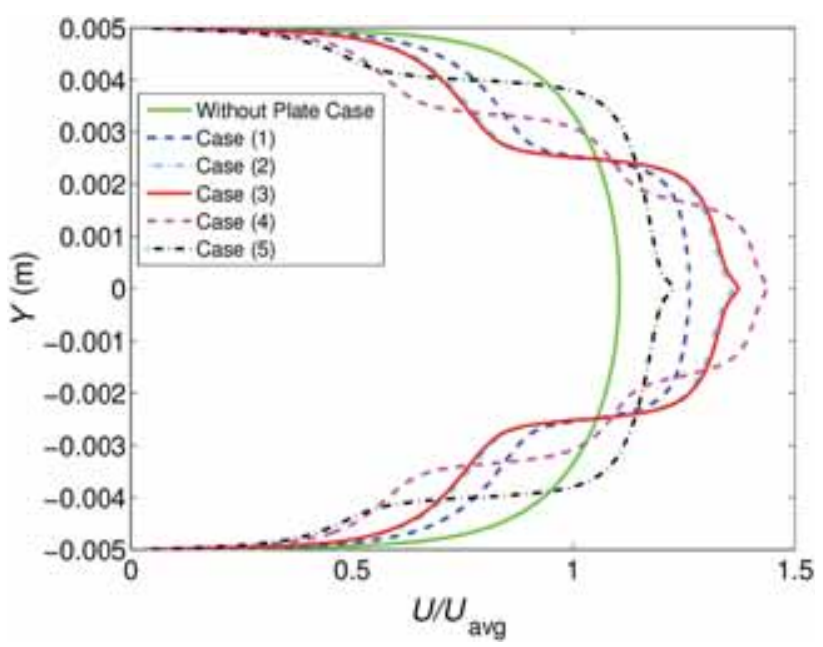

Figure 22. Velocity profiles scaled by the average velocity $\left(U_{\mathrm{avg}}\right.$ $=3.0 \mathrm{~m} / \mathrm{s}$ ) at a location $0.2 \mathrm{~m}$ from the exit at Re around 30,000 for the cases considered in figure 3 and table 4 (cases 1-5).

and also the extent of the change of the profiles. Conversely, halving $h$, doubling $U_{\text {avg }}$ and keeping $L$ and the viscosity the same would double the ratio $\left(L / U_{\text {avg }}\right) /\left(h / u^{\prime}\right)$. Thus the resident time would increase relative to the turbulent time scale and hence increase the drag reduction and also the extent of the change of the profiles. This is clearly seen in figures 20 and 22. The decreased effect of SFSes is seen as decreased peakiness of the profiles in figure 20 ( $U_{\mathrm{avg}}=0.75 \mathrm{~m} / \mathrm{s}$, half as before), while the increased effect of SFSes is seen in figure $22\left(U_{\mathrm{avg}}=3.0 \mathrm{~m} / \mathrm{s}\right.$, double as before). The corresponding changes in the turbulent kinetic energy are seen in figures 21 and 23. Table 4 summarises 


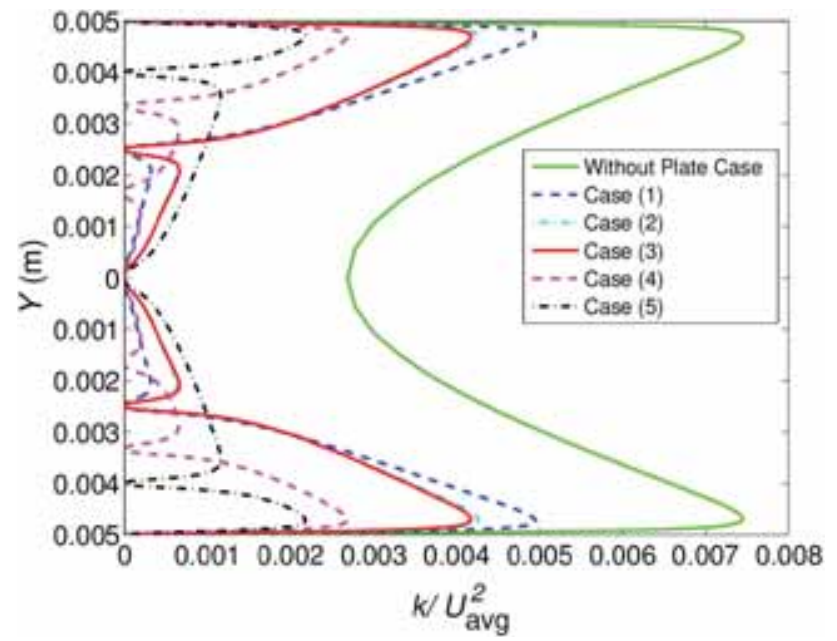

Figure 23. Turbulent kinetic energy scaled by $\left(U_{\text {avg }}\right)^{2}$ at a location $0.2 \mathrm{~m}$ from the exit at $\mathrm{Re}$ around 30,000 for the cases considered in figure 3 and table 4 (cases $1-5$ ), $U_{\text {avg }}=3.0 \mathrm{~m} / \mathrm{s}$.

the variation in drag reduction at $\operatorname{Re}=30,000$ for the five cases considered.

Looking at figures 9 and 11 , it is seen that the peak in the kinetic energy profile has moved closer to the wall. This can be associated with the shifting of the locations for the peak production of kinetic energy and transport from that location. It is thus optimal to place the SFS in this location. The position of the SFSes given in table 4 thus is more favourable to $\operatorname{Re} 10,000$ case when compared with $\operatorname{Re}$ 30,000 case. This effect cannot be investigated with RANS simulations; only LES or DNS data can be used for this purpose.

\section{Conclusion}

A novel technique of drag reduction in turbulent flows involving large scales has been presented and analysed using two-dimensional RANS simulations. The effect involves the attenuation of the lateral transport of momentum (normal to SFSes) due to kinematic wall blocking, which is brought about because of the presence of SFSes. The large scales of the turbulent flow are affected. The phenomenon is confirmed to be governed by a 'slow' turbulent time scale. The efficacy of the method is seen to depend on the ratio of two time scales - an advection time scale indicating the 'resident time' near an SFS, and the turbulent time scale. The effectiveness of the approach is also improved by judicious placement of multiple SFSes in the flow - the amount of drag reduction increases as the number of SFSes increases. The drag reduction is accompanied by significant reduction in the turbulent kinetic energy in the flow.

Three-dimensional RANS simulations would help optimise the shape, size and placement of the SFSes for improved drag reduction. Such simulations are ongoing. The understanding of the physics of the phenomenon is possible only when the transport terms of energy and the Reynolds stresses can be studied. This is possible only with LES or DNS studies involving single and multiple SFSes, which are planned.

Table 4. Summary of simulations at $\operatorname{Re}=30,000$, showing the effect of the ratio of resident to turbulent time scales.

\begin{tabular}{|c|c|c|c|c|c|c|}
\hline $\begin{array}{l}\text { Sl. } \\
\text { no. }\end{array}$ & $\begin{array}{l}\text { Simulations done (with shear-free surfaces introduced along the centreline } \\
\text { as well as along the height of the channel and simulated as fixed mesh } \\
\text { problems) }\end{array}$ & $\begin{array}{c}\text { Case A } \\
V=1.5 \\
\mathrm{~m} / \mathrm{s}\end{array}$ & $\begin{array}{l}\text { Case B } \\
V=0.5 \mathrm{~m} / \\
\mathrm{s}\end{array}$ & $\begin{array}{c}\text { Case C } \\
V=0.05 \\
\mathrm{~m} / \mathrm{s}\end{array}$ & $\begin{array}{c}\text { Case D } \\
\begin{array}{c}V=3 \\
\mathrm{~m} / \mathrm{s}\end{array}\end{array}$ & $\begin{array}{c}\text { Case E } \\
V=0.75 \mathrm{~m} / \mathrm{s}\end{array}$ \\
\hline 1 & $\begin{array}{c}3 \text { plates (length of each plate }=0.5 \mathrm{~m} \text { ) placed symmetrically in } 3 \text { rows and } \\
1 \text { column inside the channel at } 0.1 \mathrm{~m} \text { from the exit of the channel. } \\
\text { (figure } 3 \mathrm{a} \text { ) }\end{array}$ & 0.40 & 0.40 & 0.40 & 4.86 & $\begin{array}{l}-3.08 \\
\text { Increase } \\
\text { in drag }\end{array}$ \\
\hline 2 & $\begin{array}{l}6 \text { plates (length of each plate }=0.5 \mathrm{~m} \text { ) placed symmetrically in } 3 \text { rows and } \\
\qquad 2 \text { columns inside the channel. (figure } 3 \mathrm{~b} \text { ) }\end{array}$ & 4.92 & 4.92 & 4.92 & 15.40 & $\begin{array}{l}-3.18 \\
\text { Increase } \\
\text { in drag }\end{array}$ \\
\hline 3 & $\begin{array}{l}9 \text { plates (length of each plate }=0.5 \mathrm{~m} \text { ) placed symmetrically in } 3 \text { rows and } \\
\qquad 3 \text { columns inside the channel. (figure } 3 \mathrm{c} \text { ) }\end{array}$ & 11.60 & 11.59 & 11.60 & 26.67 & $\begin{array}{l}-1.49 \\
\text { Increase } \\
\text { in drag }\end{array}$ \\
\hline 4 & $\begin{array}{c}15 \text { plates placed (length of each plate }=0.5 \mathrm{~m} \text { ) placed symmetrically in } 5 \\
\text { rows and } 3 \text { columns inside the channel. (figure } 3 \mathrm{~d} \text { ) }\end{array}$ & 24.60 & 24.58 & 24.60 & 39.42 & 4.47 \\
\hline 5 & $\begin{array}{c}9 \text { plates placed in } 3 \text { rows and } 3 \text { columns with } 1 \text { row placed at } 1 \mathrm{~mm} \text { from } \\
\text { the upper wall, } 1 \text { row placed at } 1 \mathrm{~mm} \text { from the lower wall and } 1 \text { row at } \\
\text { the middle of the channel. (figure } 3 \mathrm{e} \text { ) }\end{array}$ & 50.93 & 50.92 & 50.93 & 49.38 & 49.48 \\
\hline
\end{tabular}

The ratio $\left(\left(L / U_{\text {avg }}\right) /\left(h / u^{\prime}\right)\right)$ remains constant for cases A-C, and the drag reduction and the profiles remain identical. The ratio doubles for case $\mathrm{D}$, increasing the drag reduction, and the ratio is halved for case $\mathrm{E}$, reducing the drag reduction 
It can be concluded that drag reduction involving the introduction of SFSes at the large scales of turbulent flows is a viable approach.

\section{References}

[1] Lumley J and Blossey P 1998 Control of turbulence. Annu. Rev. Fluid Mech. 30: 311-327

[2] Karniadakis G E and Choi K S 2003 Mechanisms on transverse motions in turbulent wall flows. Annu. Rev. Fluid Mech. 35: 45-62

[3] Berger T, Kim J, Lee C and Lim J 2000 Turbulent boundary layer control utilizing the Lorentz force. Phys. Fluids 12(3): 631-649

[4] Crawford C H and Karniadakis G E 1997 Reynolds stress analysis of EMHD-controlled wall turbulence, part I: streamwise forcing. Phys. Fluids 9(3): 788-806

[5] Osullivan P L and Biringen S 1998 Direct numerical simulations of low Reynolds number turbulent channel flow with EMHD control. Phys. Fluids 10(5): 1169-1181

[6] Sahlin A, Johansson A V and Alfredsson P H 1988 The possibility of drag reduction by outer layer manipulators in turbulent boundary layers. Phys. Fluids 31(10): 2814-2820

[7] Kim J and Bewley T R 2007 A linear systems approach to flow control. Annu. Rev. Fluid Mech. 39: 383-417

[8] Bushnell D M, Hefner J N and Ash R L 1977 Effect of compliant wall motion on turbulent boundary layer. Phys. Fluids 20(10): S31-S48 (part II)

[9] Christodoulou C, Liu K N and Joseph D D 1991 Combined effects of riblets and polymers on drag reduction in pipes. Phys. Fluids A Fluid Dyn. 3(5): 995-996

[10] Ferrante A and Elghobashi S 2004 On the physical mechanism of drag reduction in a spatially developing turbulent boundary layer laden with microbubbles. J. Fluid Mech. 503: 345-355

[11] Pal S, Deutsch S and Merkle C L 1989 A comparison of shear stress fluctuation statistics between microbubble modified and polymer modified turbulent boundary layers. Phys. Fluids A 1(8): 1360-1362

[12] Sanders W C, Winkel E S, Dowling D R, Perlin M and Ceccio S L 2006 Bubble friction drag reduction in a highReynolds-number flat-plate turbulent boundary layer. $J$. Fluid Mech. 552: 353-380

[13] Xu J, Maxey M R and Karniadakis G E 2002 Numerical simulation of turbulent drag reduction using micro-bubbles. J. Fluid Mech. 468: 271-281
[14] Ogata S and Watanabe K 2002 Limiting maximum dragreduction asymptote for the moment coefficient of a rotating disk in drag-reducing surfactant solution. J. Fluid Mech. 457: 325-337

[15] Pashkewitz J S, Dubief Y, Dimitropoulos D, Shaqfeh E S G and Moin P 2004 Numerical simulation of turbulent drag reduction using rigid fibres. J. Fluid Mech. 518: 281-317

[16] Oldroyd J G 1949 In Proceedings of the International Congress on Rheology, North-Holland, Amsterdam, sec. II, p. 130

[17] Toms B A 1949 In Proceedings of the International Congress on Rheology, North-Holland, Amsterdam, sec. II, p. 135

[18] Lumley J L 1969 Drag reduction by additives. Annu. Rev. Fluid Mech. 1: 367-384

[19] Berman N S 1978 Drag reduction by polymers. Annu. Rev. Fluid Mech. 10: 47-64

[20] Sreenivasan K R and White C M 2000 The onset of drag reduction by dilute polymer additives, and the maximum drag reduction asymptote. J. Fluid Mech. 409: 149-164

[21] Liberzon A, Guala M, Kinzelbach W and Tsinober A 2006 On the turbulent kinetic energy production and dissipation in dilute polymer solutions. Phys. Fluids 18(125101): 1-12

[22] Cholemari M R and Srinivasan B 2011 Investigation of novel drag reduction strategies. Project report submitted to AR\&DB, May 2011

[23] Lal S 2012 Flux enhancement by shear-free surfaces in a turbulent convection. MTech. Thesis, Department of Applied Mechanics, IIT, Delhi

[24] Majhi N K 2012 Drag reduction by modification of large scales of an internal turbulent flow. MTech. Thesis, Department of Applied Mechanics, IIT, Delhi

[25] RajaVamsi G 2009 Drag reduction by modifying the large scales of a turbulent channel flow. MTech. Thesis, Department of Applied Mechanics, IIT, Delhi

[26] Sahoo T 2010 Drag reduction by modifying the large scales of a turbulent flow. MTech. Thesis, Department of Applied Mechanics, IIT, Delhi

[27] Cholemari M R and Arakeri J H 2005 Experiments and a model of turbulent exchange flow in a vertical pipe. Int. J. Heat Mass Transf. 48(21): 4467-4473

[28] Cholemari M R and Arakeri J H 2009 Axially homogeneous, zero mean flow buoyancy-driven turbulence in a vertical pipe. J. Fluid Mech. 621: 69-102

[29] Menter F R 1994 Two-equation eddy-viscosity turbulence models for engineering applications. AIAA J. 32: 1598-1605

[30] Pope S B 2000 Turbulent flows. Cambridge University Press 